\title{
TRAFFIC MODEL OF A MICROREGION
}

\author{
Jitka Homolová ${ }^{*, * *}$ Ivan Nagy ${ }^{*, * *}$ \\ * Faculty of Transportation Sciences, CTU Prague \\ Konviktská 20, 11000 Prague 1, Czech Republic \\ ** Adaptive Systems Department, \\ Institute of Information Theory and Automation \\ P.O.Box 18, 18208 Prague 8, Czech Republic
}

\begin{abstract}
This paper introduces a new concept of the state model of one traffic microregion based on a maximum utilization of information from all measured traffic variables. The aim of the model is to estimate length of queues that are formed on arms of junctions with traffic lights. This task is trivia in case of complete knowledge of all measured traffic quantities for all junction arms. Then the model only counts simply the queue length from input and output intensities. However, the net of all needed detectors is not usually complete and some significant traffic flows (parking cars, etc.) are not measurable in practice. The model estimates the queue length in this case. In the end of the paper,the model and estimation algorithm is tested for several types of disturbances which can arise in reality. At least partially, these experiments illustrate the functionality and effectiveness of the proposed model for estimating queue lengths on the junction arms in the real traffic. Copyright ( 2005 IFAC
\end{abstract}

Keywords: traffic microregion, queue length model, Kalman filter.

\section{INTRODUCTION}

The traffic and its control are an inseparable components of all big cities in these days. Moreover, the quality of the traffic control impacts various human activities and it can be considered as one of the faces of the total society adulthood. That is why the advanced control methods have to take note not only of demands of direct users but they should also take into account their impacts on indirect users, negative environmental impacts, etc. (Kratochvílová, 2003) that are caused by the users themselves (an individual approach, bad technical condition of vehicles, etc.) or bad technical conditions of traffic infrastructure (including nonefficient traffic control).

It is very hard and long-term goal to raise people in order to be more responsive and it is still more and more complicated and expensive to magnify the existing traffic infrastructure, mainly in the historic city centers. The way how to decrease congestions during the peak hours is to apply a proper control via traffic signal lights. Then the passage time through a traffic area and the lost time spent by queuing can be reduced. It brings strong positive environmental impacts and improving the quality of a daily human life (Kratochvílová, 2003). There are many control systems for urban traffic (Papageorgiou, 2003) but the universal and definitive solution was not still found because of the stochastic and area depended nature of the traffic.

The aim of this paper is to summarize the main knowledge about the traffic flow behavior and to design the concept of the traffic model suitable for the traffic flow control. The basic principle of such 
control is traditionally minimization of the lost times, the passage times and the number of stopping during a journey. All these characteristics are proportional to the queue length (Diakaki, 2002).

Usually, traffic flow models form time course of velocity or density in dependence on intensity. Due to their general relations, such models are non-linear (Papageorgiou, 2003). In case of the urban traffic, it is also possible to model the number of vehicles within a link in dependence of inflows and outflows of the link as it was done, for example, in TUC (traffic-responsive urban control) strategy (Diakaki, 2002). The model also uses local occupancy measurements for the (nonlinear) approximation of those numbers of vehicles. This strategy uses non-linear optimization criterion for minimization and balancing of the relative occupancies of the network links.

The suggested model describes the queue length in a linear way and it is supposed to be used for traffic control which minimizes the weighted sum of queue lengths on all junction arms. The proposed model counts and estimates the queue length on the basis of maximum given traffic information. This task is trivia in case of complete knowledge of all measured traffic quantities for all junction arms. Then the model simply counts the queue length from known input and output intensities. However, the net of all needed detectors is not usually complete and some significant traffic flows (parking cars, etc.) are not measurable in practice. In this case, the model estimates the queue length relative to modelled and estimated traffic characteristics.

\section{TRAFFIC DATA}

The basic data that are necessary for an on-line traffic flow control are measured by traffic detectors. These devices can measure several traffic characteristics:

Occupancy: determines the relative time of the detector activation during the sample period, i.e. the proportion of time when the detector has been occupied and the total time of measuring period. The occupancy unit is [\%].

Intensity: denotes the number of vehicles which have passed a detector during the sample period. Usually, the value of this quantity is transformed into an hourly intensity of unit vehicles, i.e $[u v / h]$.

Velocity: can be point or segmental. It determines the average speed of vehicles passing over a detector or a certain stage. Its unit is $[\mathrm{km} / \mathrm{h}]$.
Density: denotes the number of vehicles on some road segment and its meaning comes near to the occupancy one, on condition of low velocities particularly. Its unit is $[u v / k m]$.

It is necessary to measure two variables at least to determine the actual traffic situation. The standard outputs of the measurement are values of the intensity and occupancy. The intensity denotes the queue dynamics in a sense of the queue protraction - it can be determined from it how many vehicles is captured in the queue. It is very important for us from a counting point of view but it does not fully determine the actual situation because of quadratic relation of intensity and density.

The occupancy has the similar meaning as the density and moreover, it is usually measured quantity. The higher density decreases the vehicles velocity and the intensities and queues are formed on the arms. That is why the value of the occupancy of the detectors under the queue increases. Conversely, the vehicles can go through faster in case of low traffic and they loose the minimum time by queueing - the occupancy decreases.

\section{QUEUE LENGTH}

The main problem of the task is the fact that the queue length is not measurable in practice. For our purpose, we suppose the linear relation between the occupancy and the queue length - the value of the occupancy is higher for the longer queue because the vehicles pass the detector more slowly near to the end of this queue.

Note 1. The queue represents amount of vehicles at the end of a red time.

We consider following relation:

$$
O_{t+1}=\kappa_{1} \xi_{t}+\kappa_{0}
$$

where

$$
\begin{array}{ll}
O_{t+1} & \text { is occupancy; } \\
\xi_{t} & \text { queue length; } \\
\kappa_{0}, \kappa_{1} & \text { parameters; } \\
t & \text { time instant. }
\end{array}
$$

Constants $\kappa_{0}$ and $\kappa_{1}$ can be determined experimentally for each set of the subsequent detectors because distances between the detectors placed on one approach specify the minimum and maximum queue lengths and corresponding limit average values of the occupancy can be measured. Assuming the linear relation, then the constants can be specified. In this paper, we suppose the parameters of occupancy dependance to be known and constant in time but they can also be changed continuously (known, time-dependent) or estimated (unknown, variable). 
Note 2. For measurements mentioned above, a pair of remote and strategic detectors are supposed; the detectors on stop-lines are not suitable.

\section{STATE SPACE MODEL}

We consider the queue length and the occupancy of each junction approach to be the basic state quantities for fully expressed traffic situation at given time instant. For simplicity, the state space model is derived for the junction with four arms. Each arm has only one input and output lane and all drive directions are allowed. The arms are marked by numbers from 1 to 4 anticlockwise, starting from left. The signal scheme is composed for two stages. Finally, we suppose one input and output detectors for each arm.

\subsection{Passage and queue indicator}

Passage (an amount of vehicles that pass the junction) from the given arm depends on the actual input intensity, the actual queue length on this arm and the actual setting of the traffic lights. The structural arrangement of the junction (or the corresponding quantity of the saturation flow) and the actual control (or the relative duration of the green signal) determine the capacity of a given arm, i.e. the maximum number of vehicles that can pass safely the junction during the green light. According to initial traffic conditions, a passage through the junction can be the capacity or less.

There is a condition of the passage:

$$
\begin{aligned}
I_{i ; t} z_{i ; t}+\xi_{i ; t} & \leq K_{i ; t}=S_{i} z_{i ; t} \Rightarrow \\
\Rightarrow & P_{i j ; t}=\alpha_{i j}\left(I_{i ; t} z_{i ; t}+\xi_{i ; t}\right) ; \\
I_{i ; t} z_{i ; t}+\xi_{i ; t} & >K_{i ; t}=S_{i} z_{i ; t} \Rightarrow \\
& \Rightarrow P_{i j ; t}=\alpha_{i j} K_{i ; t} .
\end{aligned}
$$

where

$$
\begin{array}{ll}
I_{i ; t} & \text { is input intensity of arm } i \\
z_{i ; t} & \text { relative green for arm } i \\
\xi_{i ; t} & \text { length of the queue on arm } i \\
K_{i ; t} & \text { capacity of arm } i ; \\
S_{i} & \text { saturation flow of arm } i \\
P_{i j ; t} & \text { passage through the junction from } \\
& \text { arm } i \text { to arm } j ; \\
\alpha_{i j} & \text { direction coefficient,i.e. the ratio of } \\
& \text { vehicles going from arm } i \text { to arm } j .
\end{array}
$$

The first case (2) arises when there is no queue (or it is small enough) at the beginning of the green and the input intensity is also small enough. On the other hand, the condition (3) is fulfilled in case of the long queues and/or high intensities.
Note 3. Our model is supposed to work with average quantities per sampling period and the queue lengths are considered in the end of this period. If some arm has its right of way during the several stages of the cycle, the passage must be calculated for all corresponding stages in case of (3).

Let's define queue indicator $\delta_{i ; t}$, which determines if there is supposed some queue on the given arm $i$ in the end of the green signal. The indicator is defined like the conventional $\delta$-function:

$\delta_{i ; t}=\left\{\begin{array}{l}1 \text { when the condition (3) is fulfilled; } \\ 0 \text { otherwise }\end{array}\right.$

The passage from the arm $i$ to the arm $j$ can be rewritten this way:

$$
P_{i j ; t}=\alpha_{i j}\left[\left(1-\delta_{i ; t}\right)\left(I_{i ; t} z_{i ; t}+\xi_{i ; t}\right)+\delta_{i ; t} K_{i j ; t}\right] .
$$

\subsection{State equation}

The state vector includes all intensities and occupancies of each input arm and it is composed arm by arm. For the mentioned junction, it is the following vector:

$$
x_{t}=\left[\xi_{1 ; t} ; O_{1 ; t} ; \xi_{2 ; t} ; O_{2 ; t} ; \xi_{3 ; t} ; O_{3 ; t} ; \xi_{4 ; t} ; O_{4 ; t}\right]^{\prime} .
$$

For the equation describing the queue length time course, the following idea is used: the new queue is the old queue plus the vehicles driving in minus vehicles driving out. Using this idea and the relation (5) for the passage, we obtain the following queue time course:

$$
\begin{aligned}
\xi_{i ; t+1}= & \delta_{i ; t} \xi_{i ; t}-\left[\left(1-\delta_{i ; t}\right) I_{i ; t}+\delta_{i ; t} S_{i ; t}\right] z_{i ; t}+ \\
& +I_{i ; t}
\end{aligned}
$$

Note 4. In case of $\delta_{i ; t}=0$, the determined relation for queue length is $\left(1-z_{i ; t}\right) I_{i ; t}$. It corresponds with the fact that the queues are formed only during the time of no right of way in case of small input (2).

Applying the equation (1) and (7) to each junction arm, we can determine the matrix state equation for the whole junction:

$$
x_{t+1}=A_{t} x_{t}+B_{t} z_{t}+F_{t}+e_{t},
$$

where

$x_{t} \quad$ is state vector;

$A_{t} \quad$ time variant matrix of state dependence parameters;

$B_{t} \quad$ time variant matrix of passages;

$z_{t} \quad$ vector of the relative greens;

$F_{t} \quad$ time variant matrix of constants;

$e_{t} \quad$ process noise. 


\subsection{Output equation}

For maximum utilizing actual traffic data and correcting the state estimates, we suppose to use Kalman filter (Peterka, 1981). First, it is necessary to select all quantities which can be measured and compared to predicted ones. Thus we can use only occupancies of input detectors and intensities of output detectors (passage intensity) for correcting the whole state vector. Supposing the input and output detector for each junction arm, the modelled output is

$$
y_{t}=\left[y_{1 ; t} ; y_{2 ; t} ; y_{3 ; t} ; y_{4 ; t} ; O_{1 ; t} ; O_{2 ; t} ; O_{3 ; t} ; O_{4 ; t}\right]^{\prime} \text {, }
$$

where

$y_{i ; t} \quad$ is measured output of arm $i$;

$O_{i ; t} \quad$ occupancy of the input detector on $\operatorname{arm} i$.

First, we determine the total outputs (the passages) into the $\operatorname{arm} i$, i.e. the sum of the individual arm passages that head towards the given arm $i$. We use the knowledge of the direction coefficients (the ratios of the turning vehicles).

Note 5. Let's recollect the basic properties of the direction coefficients: $\sum_{j} \alpha_{i j}=1 \& \alpha_{i i}=0, \forall i=$ $1,2,3,4$.

According to the relation (5), the arm output like the sum of the passages from the remaining arms is

$y_{i ; t}=\sum_{j} \alpha_{j i}\left[\left(1-\delta_{j ; t}\right)\left(\xi_{j ; t}+I_{j ; t} z_{j ; t}\right)+\delta_{j ; t} S_{j} z_{j ; t}\right]$.

For determining the matrix output equation, the modified equation (10) end the identity equation of the occupancy

$$
\begin{aligned}
y_{i ; t} & =\sum_{j}\left[\alpha_{j i}\left(1-\delta_{j ; t}\right) \xi_{j ; t}+\right. \\
\left.O_{j i}\left[\left(1-\delta_{j ; t}\right) I_{j ; t}+\delta_{j ; t} S_{j}\right] z_{j ; t}\right] & \\
O_{i ; t} & =O_{i ; t},
\end{aligned}
$$

applied for each arm with the input and output detector. The matrix output equation then is

$$
y_{t}=C_{t} x_{t}+D_{t} z_{t}+\epsilon_{t},
$$

where

$\begin{array}{ll}x_{t} & \text { is state vector; } \\ C_{t} & \text { time variant matrix of state depen- } \\ & \text { dence parameters; } \\ D_{t} & \text { time variant matrix of passages with } \\ & \text { respect to drive directions; } \\ z_{t} & \text { vector of the relative greens; } \\ \epsilon_{t} & \text { measurement noise. }\end{array}$

All matrices of the mentioned state space model are supposed to be automatically generated for any set of junctions on the basis of traffic data measurement at disposal.

\section{ESTIMATION OF STATES}

It is possible to measure some of the modelled quantities continuously and we use this fact for the specification of the estimated values. The modelled quantities can be considered as random variables and then their estimates at the given time instant are the point estimates of these random variables in a mathematical statistics sense and with the utilization of their conditional probability densities.

Applying the Bayesian approach, the given estimation task can be solved as the algebraic recursion that already works with conditional mean values and covariances only. This method is generally known as a Kalman filter (Peterka, 1981):

$$
\begin{aligned}
\hat{y}_{t \mid t-1} & =C \hat{x}_{t \mid t-1}+D z_{t} \\
\hat{R}_{y_{t \mid t-1}} & =R_{\epsilon}+C S_{t \mid t-1} C^{T} \\
S_{t \mid t} & =S_{t \mid t-1}-S_{t \mid t-1} C^{T} \hat{R}_{y_{t \mid t-1}}^{-1} C S_{t \mid t-1} \\
\hat{e}_{t} & =y_{t}-\hat{y}_{t \mid t-1} \\
\hat{x}_{t \mid t} & =\hat{x}_{t \mid t-1}+S_{t \mid t} C^{T} R_{\epsilon}^{-1} \hat{e}_{t} \\
\hat{x}_{t+1 \mid t} & =A \hat{x}_{t \mid t}+B z_{t} \\
S_{t+1 \mid t} & =R_{e}+A S_{t \mid t} A^{T}
\end{aligned}
$$

where

$R_{e}, R_{\epsilon} \quad$ are covariance matrices.

Prediction of the output is compared with the measured output and the prediction error $\hat{e}_{t}$ is used for update of the estimated state. In this way, the correction of state estimates can be done using all obtained measurements of the occupancies and the intensities by the input and output detectors.

\section{EXPERIMENTS}

The described model for one four-armed junction is used together with the Kalman filtering for few experiments. For the mere verification of the suggested traffic model, the simple fixed control is applied. There is the constant number of the stages and their invariable relative green times for the whole time.

For preservation of the traffic nature of the task, the real traffic data from certain real area were used for the experiments. This measurement was not made for our needs so we were forced to use the segment of this data, actually the intensities 
only, for the simulation of the occupancies and the queue lengths. The prior data represented one day measurement, i.e. 96 measurements in 15 minutes sample period.

\subsection{Simulation}

The queue length simulation was based on a pure addition of the incoming intensity to the queue length and subtracting the actual passages. Based on the real course of the intensities, these simulated courses of the queue lengths approximately correspond with their real course.

The occupancies were simulated due to the simulated queue lengths and the assumed linear relation between them (1). Finally, we computed the output intensities of all arms.

\subsection{Modelling}

The quantities usable after the simulation are the input intensities (real, measured), the input occupancies (simulated) and the output intensities (simulated) for each junction arm. All these traffic characteristics are used for the modelling and the estimating due to (8), (12) and (13).

In case of the availability of all required data without the major errors and the availability of parameter values that are near to the real, actually simulated values, the model only recalculates the input variables deterministically. The estimates of queue lengths then correspond with the simulated ones and the deviations are minimal. This fact just approves for the correct design of the traffic model.

For that reason, the following experiments aim at the testing of the estimation algorithm (the Kalman filter) effectiveness. These experiments partially approve for the possibility of practical use of the proposed model, at least.

\subsection{Experiment 1 - Memory defect}

In the first experiment, the memory defect of all input arms is simulated during the relatively long time - for 11 sample periods, i.e. approximately for 3 hours. There is available incorrect state information between 50th and 61st time instant and the model works with the zero values of the queues.

The steep decrease of the queue length values can be seen during the defect time on the fig. 1 but the algorithm tries to balance the differences as soon as possible. This process is very well pointed at the course of the prediction errors of

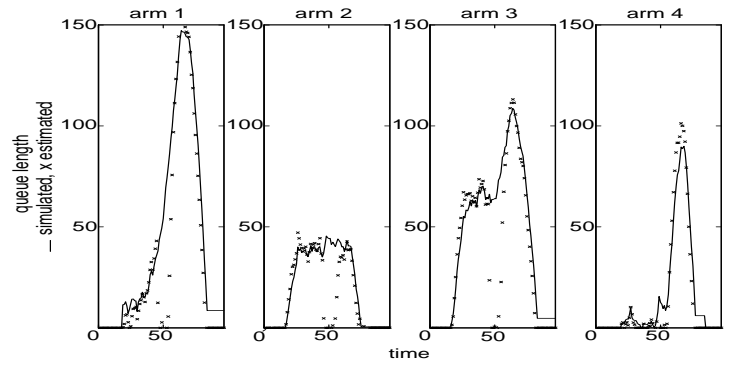

Fig. 1. Experiment 1: Memory defect
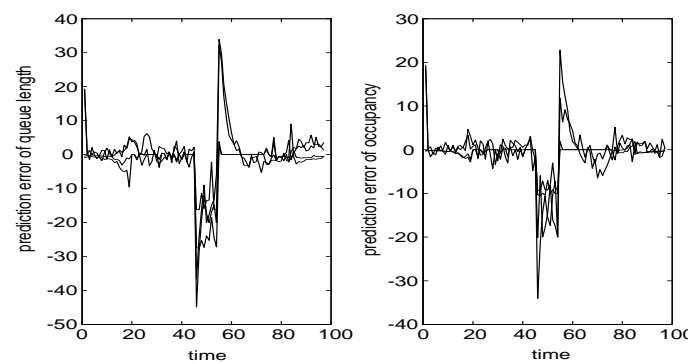

Fig. 2. Experiment 1: Prediction errors

the model outputs (see fig. 2). The modelled data soon correspond to the simulated values.

Finally, it is necessary to note that such long-term defects of memory is not very probable in practice and according to the obtained results, we expect that the smaller defects will be balanced much better and much quickly.

\subsection{Experiment 2 - Incorrect model parameter}

In case of an incorrect model parameter, computation errors are systematic. This kind of the error seems to be the most serious because of the integrative nature of the model.

The saturation flow as one of the model parameters is bound up the structural arrangement of the junction. The structure change (e.g. the change of the radius of curvature, the road gradient, the number of lanes, etc.) without the new traffic handling (the quantification of the new parameters) is impossible in practice. On the other hand, the values of the saturation flows can be temporarily changed in consequence of an accident, building works, etc. The vehicles drive through the junction much slowly (or quickly) and the number of the passing cars is smaller or bigger than expected.

We simulate the differences of the saturation flows at the range of $10 \%$. The fig. 3 shows that the quality of the queue length estimation is noticeably worse. However, the differences are satisfactory.

\subsection{Experiment 3 - Disruption of linearity}

The last experiment tests the disruption of the pure linear relation between the occupancy and 


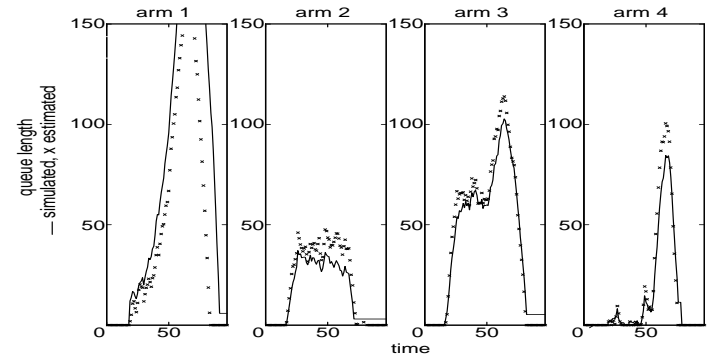

Fig. 3. Experiment 2: Incorrect model parameter

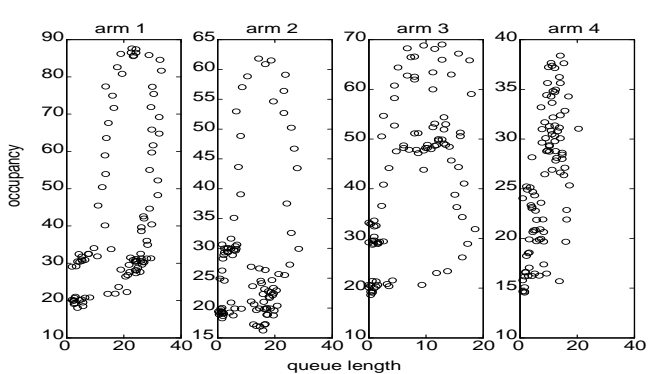

Fig. 4. Experiment 3: Disruption of linearity

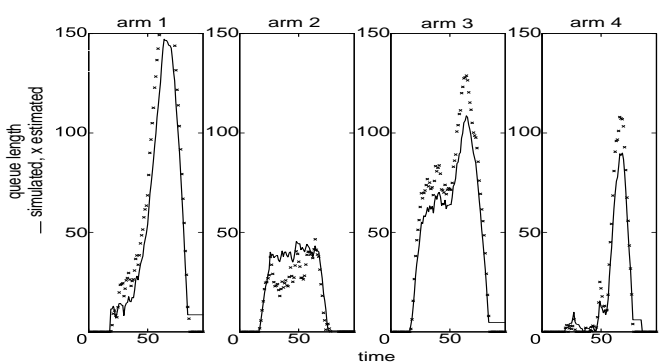

Fig. 5. Experiment 3: Queue length estimates

the queue length. This experiment is extra important because the assumption of the linearity is the ground of the proposed model. The linearity can be disrupted quite easily in practice - a bad data sample, other influences of the traffic character, etc.

The model kept the assumption of the linearity as previously but the simulation of the occupancy was different. This traffic characteristics had been also counted according to the equation 1 but the randomly generated values were added to them. The fig. 4 shows that the linear relation had been disrupted very strongly. However, the estimates of the queue lengths are relatively satisfactory yet (see fig. 5). Thus the model can manage this disruption and can be used in case of non purely linear relation between the occupancy and the queue length.

\section{CONCLUSION}

This paper demonstrates the model of the traffic micro-region which can, exploiting maximum of data measurement information, estimate the queue lengths that are created on the junction arms. It was shown that the model was observable and referred about the actual traffic situation very well. Moreover, all simulated errors and disruptions were rather too big than the real. That is why we expect the model will balance the smaller but the more real errors much better.

The derived model is used for the optimization of the green times which minimizes the weighted sum of the queue lengths of the junction. This minimization leads to the minimization of the loss time during the passage through the given microregion and to the total improvement of the actual traffic situation. If it is possible to fall back on the good estimates of the queue lengths that are not measurable yet in practice, an effective and a quality control can be applied.

\section{Acknowledgements}

This research has been partially supported by Ministry of Transportation, Czech Republic, National programme of research 2004 - 2009, Project num. 1F43A/003/120.

\section{REFERENCES}

Diakaki, Ch., Dinopoulou, V., Aboudolas, K. and Papageorgiou, M. (2002) Deliverable 9: Final System Development Report, IST-200028090. Technical University of Crete.

Kratochvílová, J. and Nagy, I. (2003) Bibliographic Search for Optimization Methods of Signal Traffic Control, UTIA-B $2003015 \%$. ÚTIA AV ČR, Praha.

Kratochvílová, J. and Nagy, I. (2004) Traffic control of microregion In: CMP'04: MULTIPLE PARTICIPANT DECISION MAKING, Theory, algorithms, software and applications (J. Andrýsek, M. Kárný and J. Kracík, ed.). Advanced Knowledge International, Adelaide, Australia, pp. 161-171.

Papageorgiou, M., Diakaki, Ch., Dinopoulou, V., Kotsialos, A. and Wang, Y. (2003) Review of Road Traffic control Straegies In: Proceedings of the IEEE, Vol. 91, pp. 2043 - 2067,

Diakaki, Ch.,Papageorgiou, M. and Aboudolas, K. (2002) A multivariable regulator approach to traffic-responsive network-wide signal control In: Control Engineering Practice, Vol. 10, pp. $183-195$.

Peterka, V. (1981) Bayesian approach to system identification. In: Trends and Progress in System Identification (P. Eykhoff, ed.). Pergamon Press, Oxford, pp. 239-304. 